\title{
Can an Electric Field Induce an Antiferroelectric Phase Out of a Ferroelectric Phase?
}

\author{
X. Tan, ${ }^{1, *}$ J. Frederick, ${ }^{1}$ C. Ma, ${ }^{1}$ W. Jo, ${ }^{2}$ and J. Rödel ${ }^{2}$ \\ ${ }^{1}$ Department of Materials Science and Engineering, Iowa State University, Ames, Iowa 50011, USA \\ ${ }^{2}$ Institute of Materials Science, Technische Universität Darmstadt, 64287 Darmstadt, Germany
}

(Received 30 July 2010; published 14 December 2010)

\begin{abstract}
It has been widely accepted that electric fields favor the ferroelectric phase with parallel electric dipoles over the antiferroelectric phase. With detailed measurements in polycrystalline ceramics of $\mathrm{Pb}_{0.99} \mathrm{Nb}_{0.02}\left[\left(\mathrm{Zr}_{0.57} \mathrm{Sn}_{0.43}\right)_{1-y} \mathrm{Ti}_{y}\right]_{0.98} \mathrm{O}_{3}$, we demonstrate in this Letter that electric fields can induce an antiferroelectric phase out of a ferroelectric phase, i.e., trigger an apparently unlikely ferroelectricto-antiferroelectric phase transition. We suggest that it is caused by the volume contraction from the converse piezoelectric effect at the coercive field with a reversed polarity.
\end{abstract}

When a gaseous or liquid phase with polar molecules is placed into an electric field, the dipole moment of molecules will be aligned to the direction of the applied field due to the presence of Coulomb forces. In ferroelectric (FE) and antiferroelectric (AFE) solid crystals, the electric dipoles are often originated from cation displacements in the unit cell—parallel to each other in FE and antiparallel in AFE crystals [1,2]. Aligning originally antiparallel electric dipoles to the direction of the applied field thus requires cooperative adjustment of the crystal lattice in many unit cells and corresponds to a first order displacive phase transition [3-10].

The vast majority of AFE ceramics are based on the prototype compound $\mathrm{PbZrO}_{3}$ with the perovskite structure [3-14]. In these oxides, the AFE-to-FE phase transition manifests itself in the development of a large polarization and is generally accompanied by a significant volume expansion when the applied field exceeds a critical magnitude $E_{F}$. The developed macroscopic polarization results from the antiparallel dipoles being forced parallel at the transition. The associated volume change at the phase transition implies that mechanical stresses are expected to strongly influence the phase transition, which has been experimentally demonstrated [15].

Furthermore, compressive stresses have been observed to trigger the FE-to-AFE phase transition in FE ceramics with compositions close to the AFE/FE phase boundary $[16,17]$. It is of highly scientific curiosity and technological significance to clarify whether the electric field, which is widely believed to favor the FE phase over the AFE one, can also induce an AFE phase out of a FE phase.

In the present work, the occurrence of such a transition, which is seemingly against the Coulomb law, is confirmed in polycrystalline ceramics at room temperature with experimental evidences from strain measurements at the macroscopic scale and in situ $\mathrm{x}$-ray diffraction at the microscopic scale. The strategy takes advantage of the fact that a poled FE ceramic contracts in volume when electric fields with a reversed polarity are applied and reaches a minimum volume around the coercive field $-E_{c}$ $[12,18]$. Since the compressive stress-induced FE-to-AFE phase transition is triggered by a critical volume contraction in FE oxides [16,17], it is conceived that the electric field-induced FE-to-AFE phase transition may also be realized and mediated by the volume contraction at $-E_{c}$ if the composition of the ceramic is properly tuned.

The search for the AFE/FE phase boundary composition is focused on the $\mathrm{Pb}_{0.99} \mathrm{Nb}_{0.02}\left[\left(\mathrm{Zr}_{0.57} \mathrm{Sn}_{0.43}\right)_{1-y} \mathrm{Ti}_{y}\right]_{0.98} \mathrm{O}_{3}$ (abbreviated as PNZST43/100y/2 hereafter) perovskite oxide, which is one of the most studied AFE ceramic systems [8-10,15]. Ceramics with $y=0.060,0.063$, $0.067,0.069,0.071$, and 0.075 were prepared by the solid state reaction method from high purity oxides [10]. For all the measurements, the applied electric fields were along the thickness direction of thin disk samples, taking a triangular waveform with a loading rate of $1 \mathrm{kV} / \mathrm{mm}$ per second. Three linear variable displacement transducers were used to measure the electric field-induced strains; one for the longitudinal strain $x_{33}$ in the thickness direction and two for the transverse strain $x_{11}$ in the radial direction. The in situ X-ray diffraction experiment was carried out on a Siemens D500 diffractometer using $\mathrm{Cu}-K \alpha$ radiation. The data were collected in the $\theta-2 \theta$ scan mode from the electroded flat face.

In the PNZST43/100y/2 composition series, the AFE order is weakened while the FE order is strengthened with increase in $y$ ( $\mathrm{Ti}$ content). At room temperature $\left(25^{\circ} \mathrm{C}\right)$, the AFE/FE phase boundary is around $y=0.080$ [10]. Figure 1 displays the dielectric loss tangent measured during cooling and heating for PNZST43/6.0/2 and 43/7.1/2. In these ceramics, the loss tangent in the $\mathrm{FE}$ phase is greater than that in the AFE phase $[8,19]$. It is evident that, depending on thermal history, either FE or AFE ordering can dominate at room temperature $\left(25^{\circ} \mathrm{C}\right)$ in PNZST43/7.1/2. In contrast, the PNZST43/6.0/2 ceramic is always dominated by the AFE ordering at room temperature.

The transverse strain $x_{11}$, longitudinal strain $x_{33}$, and volume strain $\left(x_{33}+2 x_{11}\right)$ as a function of electric field in 


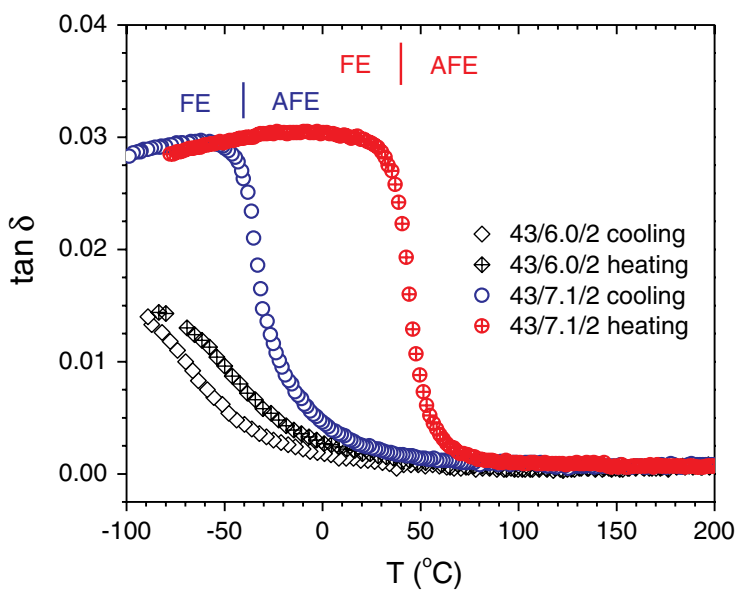

FIG. 1 (color online). Hysteresis in the dielectric loss tangent measured at $1 \mathrm{kHz}$ in PNZST43/6.0/2 and 43/7.1/2 during heating and cooling at $3{ }^{\circ} \mathrm{C} / \mathrm{min}$. As indicated by the phase regions marked in the upper portion of the figure for PNZST43/ $7.1 / 2$, either the AFE or the FE phase can be stabilized at room temperature $\left(25^{\circ} \mathrm{C}\right)$.

these two ceramics at virgin state are contrasted in Fig. 2 for the very first cycle of application. As is clear from the AFE composition PNZST43/6.0/2, $x_{11}, x_{33}$, and $\left(x_{33}+\right.$ $\left.2 x_{11}\right)$ all abruptly increase at $E_{F}$ during loading and abruptly drop at $E_{A}$ during unloading, indicating the reversible AFE-to-FE phase transition with a volume strain $\sim 0.4 \%$. For PNZST43/7.1/2, an abrupt volume expansion of $\sim 0.4 \%$ is also seen at $E_{F}$ from the AFE-to-FE phase transition [Fig. 2(d)]. However, it is noted that $x_{11}, x_{33}$, and $\left(x_{33}+2 x_{11}\right)$ do not show any abrupt changes during the process when the applied field returns to zero, indicating that the induced FE phase remains and the AFE phase is not resumed during unloading. This observation is

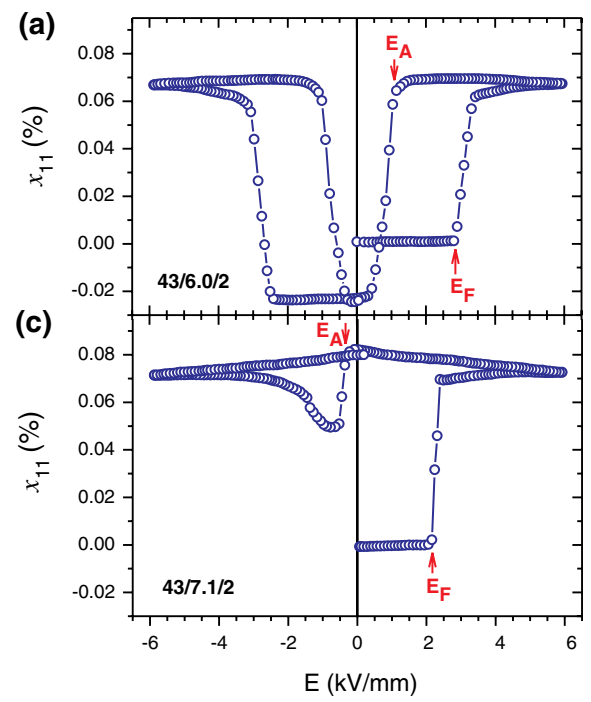

consistent with the results shown in Fig. 1. Upon reversing the polarity of the electric field in the third quarter of the cycle, a sudden drop in $x_{11}$ is observed, marking the FE-to-AFE phase transition [Fig. 2(c)]. Obviously $E_{F}$ and $E_{A}$ have the opposite sign within a given half-cycle in the composition PNZST43/7.1/2. Hence, the metastable FE phase induced by the electric field in the first quarter cycle can be transformed back to the AFE phase when a field with opposite polarity is applied. However, the resulting sudden volume reduction is $0.18 \%$ [Fig. 2(d)]. Compared with the volume expansion of $\sim 0.4 \%$ during the first quarter cycle of electric field, it appears that about half of the grains have experienced the electric field-induced FE-to-AFE phase transition.

The electric field-induced FE-to-AFE transition can be better appreciated by the trend displayed in $E_{A}$ in the $\mathrm{Pb}_{0.99} \mathrm{Nb}_{0.02}\left[\left(\mathrm{Zr}_{0.57} \mathrm{Sn}_{0.43}\right)_{1-y} \mathrm{Ti}_{y}\right]_{0.98} \mathrm{O}_{3}$ composition series. As $y$ increases, both $E_{F}$ and $E_{A}$ monotonically decrease. $E_{A}$ starts to become negative in PNZST43/6.9/2, demonstrating the occurrence of the electric field-induced FE-to-AFE phase transition (Fig. 3). However, as reflected by the magnitude of the abrupt drop in $x_{11}$, the extent of this phase transition decreases considerably with further increase in composition $y$.

Examination of the change of crystal structure under applied electric fields by x-ray diffraction further confirms the electric field-induced FE-to-AFE transition. Again, PNZST43/6.0/2 is used as a reference. Figures 4(a) and 4(b) indicate that the as-sintered ceramic displays a tetragonal $(T)$ symmetry in the virgin state consistent with previous studies in the literature [20,21]. The induced FE phase under electric fields displays a rhombohedral $(R)$ distortion of the perovskite structure [20] as confirmed by the disappearance of the $T(200) / T(002)$ splitting

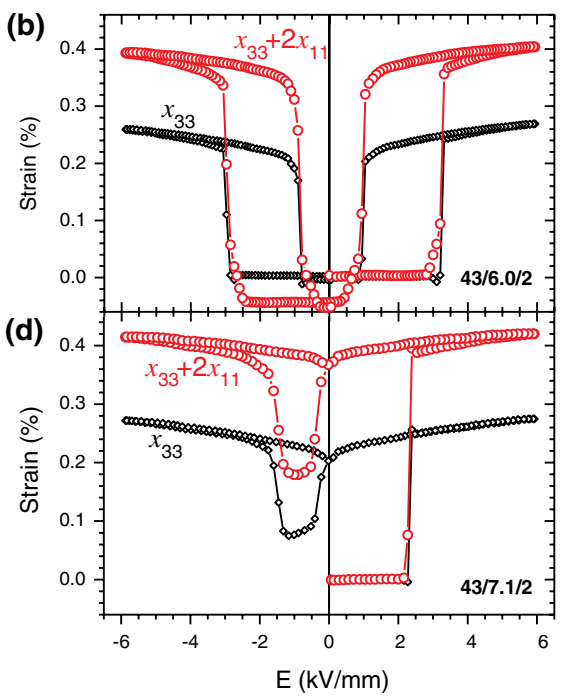

FIG. 2 (color online). The transverse strain $x_{11}$, longitudinal strain $x_{33}$, and volume strain $\left(x_{33}+2 x_{11}\right)$ developed in polycrystalline ceramics in response to the very first cycle of applied electric fields at $25^{\circ} \mathrm{C}$. (a) and (b), PNZST43/6.0/2. (c) and (d), PNZST43/7.1/2. Note that the induced FE phase in PNZST43/7.1/2 is maintained after the applied field is removed. An electric field with a reversed polarity triggers the FE-to-AFE phase transition; i.e., $E_{A}$ is negative. 


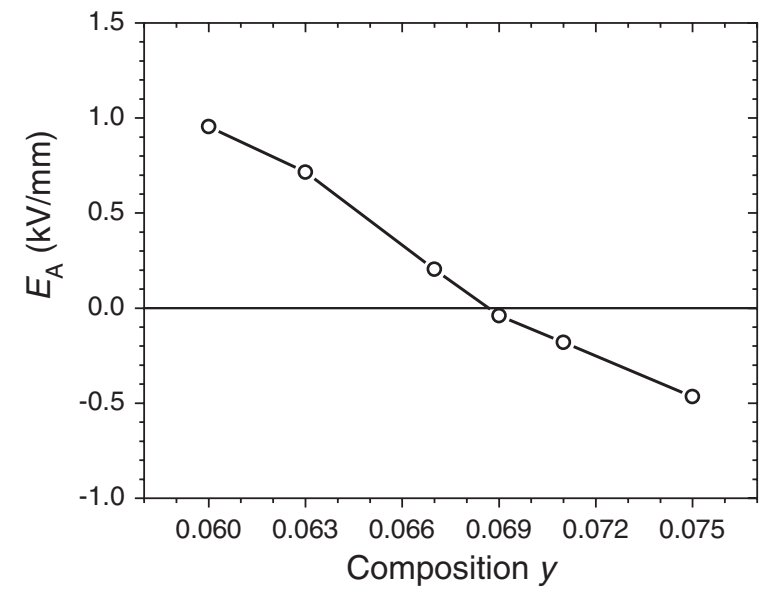

FIG. 3. The change of $E_{A}$ as a function of composition $y$ in $\mathrm{Pb}_{0.99} \mathrm{Nb}_{0.02}\left[\left(\mathrm{Zr}_{0.57} \mathrm{Sn}_{0.43}\right)_{1-y} \mathrm{Ti}_{y}\right]_{0.98} \mathrm{O}_{3}$ at $25^{\circ} \mathrm{C}$. Note that $E_{A}$ becomes negative in PNZST43/6.9/2, 43/7.1/2, and 43/7.5/2.

$\left(2 \theta \sim 44^{\circ}\right)$ and the presence of splitting of $R(222) /$ $R(\overline{2} 22)\left(2 \theta \sim 81^{\circ}\right)$ at $-4.0 \mathrm{kV} / \mathrm{mm}$ in the third quarter of the first cycle of electric field application. The calculated volume of the primitive cell (containing one $\mathrm{ABO}_{3}$ formula unit) is displayed in Fig. 4(c): $68.65 \AA^{3}$ for the tetragonal at virgin state and $69.08 \AA^{3}$ for the rhombohedral at $-4.0 \mathrm{kV} / \mathrm{mm}$. The primitive cell volume expansion of $0.6 \%$ agrees well with the macroscopic strain measurement results provided in Fig. 2(b). At the end of the first cycle where the electric field returns to zero, the AFE phase with tetragonal distortion is resumed. The disappearance of the $R(222)$ peak shown in Fig. 4(b) indicates a complete return to the AFE tetragonal phase while the weaker intensity of the $T(002)$ peak relative to that of the virgin state suggests a texture has developed [20,21]. This texture development is consistent with the nonzero $x_{11}$ at zero field after the first cycle [Fig. 2(a)]. Obviously the reappearance of the $T(002)$ peak can be considered as the signature for the AFE tetragonal phase. As such, this peak is highlighted by the dashed box in Fig. 4(a).

Figures 4(d) and 4(e) display the results of the in situ x-ray diffraction experiment on PNZST43/7.1/2. Similar to that in PNZST43/6.0/2, a tetragonal distortion in the perovskite lattice with AFE order is seen in the virgin state while the FE phase with a rhombohedral structure results under strong electric fields. When the electric field reaches zero at the end of the very first cycle, the rhombohedral FE phase is preserved. However, when an electric field with reversed polarity at a magnitude of $0.5 \mathrm{kV} / \mathrm{mm}$ is applied, the $T(002)$ signature peak reappears [highlighted by the dashed box in Fig. 4(d)], conclusively verifying the occurrence of the electric field-induced FE-to-AFE phase transition. Compared to the $\left(x_{33}+2 x_{11}\right)$ vs $E$ curve for PNZST43/7.1/2 in Fig. 2(d), the field $0.5 \mathrm{kV} / \mathrm{mm}$ is within the field range exhibiting the lowest readings (smallest volume). In addition, this field level is almost identical to the coercive field $E_{c}$ of the induced FE phase. The volume

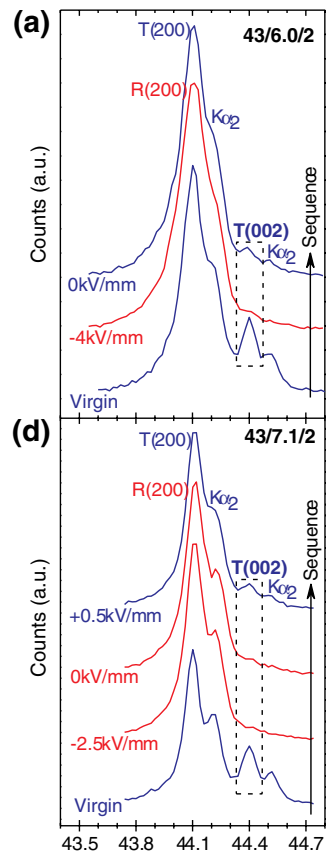

$2 \theta$

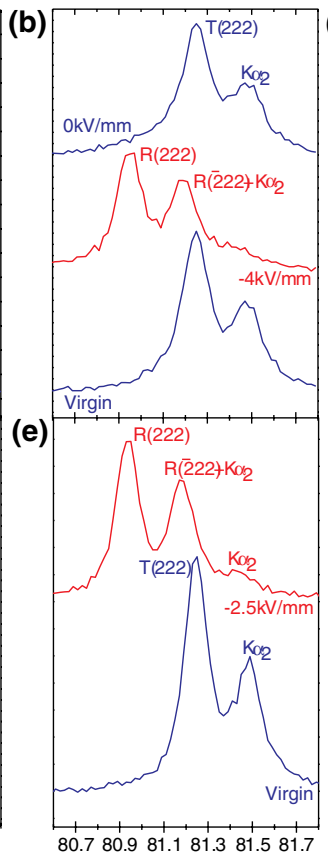

$2 \theta$
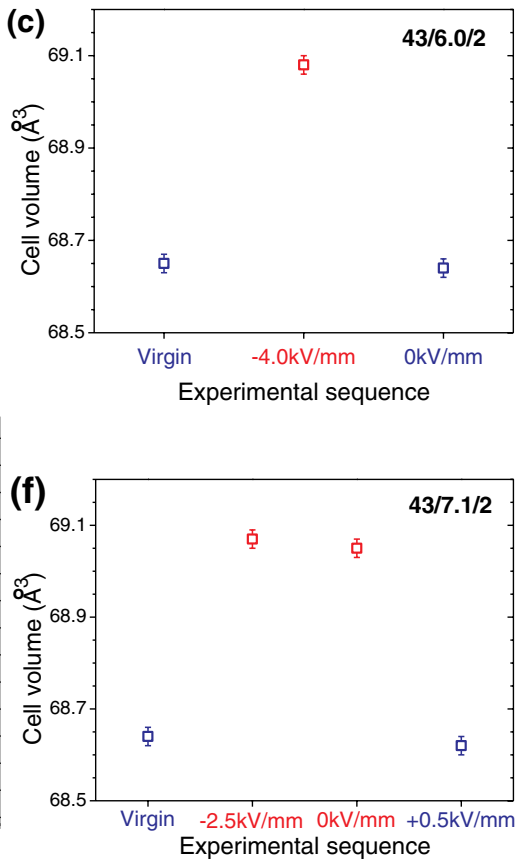

FIG. 4 (color online). The X-ray diffraction results from ceramic specimens under electric fields at $25^{\circ} \mathrm{C}$. (a) The pseudocubic $\{200\}_{c}$ peak, (b) the pseudocubic $\{222\}_{c}$ peak, and (c) the primitive cell volume of PNZST43/6.0/2. (d) The $\{200\}_{c}$ peak, (e) the $\{222\}_{c}$ peak, and (f) the primitive cell volume of PNZST43/7.1/2. The primitive cell volume is calculated from data shown in (a) and (d) for the tetragonal [at virgin and $0 \mathrm{kV} / \mathrm{mm}$ in (c) while at virgin and $+0.5 \mathrm{kV} / \mathrm{mm}$ in (f)] and the rhombohedral [at $-4.0 \mathrm{kV} / \mathrm{mm}$ in (c) and at $-2.5 \mathrm{kV} / \mathrm{mm}$ and $0 \mathrm{kV} / \mathrm{mm}$ in (f)] phases in PNZST43/6.0/2 and 43/7.1/2, respectively. 
of the primitive cell calculated from the in situ $\mathrm{x}$-ray results is displayed in Fig. 4(f) for the four conditions shown in Fig. 4(d). The volume of the tetragonal cell is provided for the virgin state and under $+0.5 \mathrm{kV} / \mathrm{mm}$ while that of the rhombohedral cell is calculated under conditions of $-2.5 \mathrm{kV} / \mathrm{mm}$ and $0 \mathrm{kV} / \mathrm{mm}$. The cell volume expansion of $0.6 \%$ from the virgin state to that under $-2.5 \mathrm{kV} / \mathrm{mm}$ is consistent with the abrupt expansion of $0.4 \%$ in macroscopic volume strain during the first quarter cycle electric field application [Fig. 2(d)].

It is suggested that the key factors for the seemingly unlikely electric field-induced FE-to-AFE phase transition are the chemical composition and the process of exposing the ceramic to an intense electric field. The composition has to be in the range where the energy barrier between the AFE and FE phases is low so that either one can be preserved at ambient conditions without any applied electric fields or mechanical stresses [5,12]. When the ceramic is exposed to an intense electric field, not only can the metastable FE phase be realized, but also the electric dipoles in the induced FE phase are forced to align along the field direction and the induced FE phase becomes piezoelectric [21]. As seen in Fig. 2(d), there is a volume contraction on the order of $0.02 \%$ in PNZST43/7.1/2 right before the field reaches $E_{A}$. It is interesting to notice that in FE $\mathrm{Pb}_{0.99}\left(\mathrm{Zr}_{0.95} \mathrm{Ti}_{0.05}\right)_{0.98} \mathrm{Nb}_{0.02} \mathrm{O}_{3}$, a compressive volume strain of $\sim 0.30 \%$ due to a $250 \mathrm{MPa}$ hydrostatic pressure was observed to trigger the FE-to-AFE phase transition with a volume reduction of $\sim 0.80 \%$ at room temperature $[16,17]$. The ceramics investigated here, especially PNZST43/7.1/2, are at the AFE/FE phase boundary at room temperature; the needed volume contraction to trigger the FE-to-AFE transition is thus significantly reduced. Furthermore, this volume contraction from the converse piezoelectric effect shows a complex distribution in polycrystalline oxides due to the anisotropy in piezoelectric strain and randomly oriented grains. Based on the fact that the volume strain is only partially recovered during the electric field-induced FE-to-AFE phase transition in PNZST43/7.1/2 at $25^{\circ} \mathrm{C}$ [Fig. 2(d)], it is speculated that only those grains with higher piezoelectric response are transformed to the AFE phase. Since in poled FE single crystals volume contraction also occurs at $-E_{c}$, it is possible that the electric field-induced FE-to-AFE phase transition observed here may occur in single crystals as well once the composition of the crystal is correctly chosen.

In summary, an electric field-induced FE-to-AFE phase transition in polycrystalline ceramics is conclusively demonstrated by macroscopic strain measurements as well as by crystal structure analysis. However, the observed FE-toAFE transition is fundamentally different from a thermodynamically equilibrium one. The situation is quite similar to a recent discovery that supercooled water on a positively charged surface starts freezing under heating [22]. Since FE and AFE materials are of technological importance for transducers [11,12], energy storage [13] and electrocaloric devices [14], this discovery may lead to the production of new electric charge storage devices.

This work was supported by the National Science Foundation (NSF) through Grant No. CMMI-1027873 and by the Deutsche Forschungsgemeinschaft (DFG) under SFB 595.

*Corresponding author. xtan@iastate.edu

[1] C. Kittel, Phys. Rev. 82, 729 (1951).

[2] E. Sawaguchi, H. Maniwa, and S. Hoshino, Phys. Rev. 83, 1078 (1951).

[3] D. Berlincourt, H. H. A. Krueger, and B. Jaffe, J. Phys. Chem. Solids 25, 659 (1964).

[4] W. Pan, Q. Zhang, A. Bhalla, and L.E. Cross, J. Am. Ceram. Soc. 72, 571 (1989).

[5] P. Yang and D. A. Payne, J. Appl. Phys. 71, 1361 (1992).

[6] H. He and X. Tan, Phys. Rev. B 72, 024102 (2005).

[7] T. Tani, J.F. Li, D. Viehland, and D. A. Payne, J. Appl. Phys. 75, 3017 (1994).

[8] D. Viehland, D. Forst, Z. Xu, and J. F. Li, J. Am. Ceram. Soc. 78, 2101 (1995).

[9] X. Tan, W. Jo, T. Granzow, J. Frederick, E. Aulbach, and J. Rödel, Appl. Phys. Lett. 94, 042909 (2009).

[10] H. He and X. Tan, J. Phys. Condens. Matter 19, 136003 (2007).

[11] B. Jaffe, W. R. Cook, and H. Jaffe, Piezoelectric Ceramics (Academic Press, London, 1971).

[12] K. Uchino, Jpn. J. Appl. Phys. 24, Suppl. 24-2, 460 (1985).

[13] J.P. Dougherty, U.S. Patent No. 5545 184, 1996.

[14] A. S. Mischenko, Q. Zhang, J. F. Scott, R. W. Whatmore, and N. D. Mathur, Science 311, 1270 (2006).

[15] X. Tan, J. Frederick, C. Ma, E. Aulbach, M. Marsilius, W. Hong, T. Granzow, W. Jo, and J. Rödel, Phys. Rev. B 81, 014103 (2010).

[16] D.H. Zeuch, S. T. Montgomery, and D. J. Holcomb, J. Mater. Res. 15, 689 (2000).

[17] M. Avdeev, J. D. Jorgensen, S. Short, G. A. Samara, E. L. Venturini, P. Yang, and B. Morosin, Phys. Rev. B 73, 064105 (2006).

[18] X. Tan, E. Aulbach, W. Jo, T. Granzow, J. Kling, M. Marsilius, H. J. Kleebe, and J. Rödel, J. Appl. Phys. 106, 044107 (2009).

[19] D. Viehland, Phys. Rev. B 52, 778 (1995).

[20] C. T. Blue, J. C. Hicks, S. E. Park, S. Yoshikawa, and L. E. Cross, Appl. Phys. Lett. 68, 2942 (1996).

[21] S. E. Park, M. J. Pan, K. Markowski, S. Yoshikawa, and L. E. Cross, J. Appl. Phys. 82, 1798 (1997).

[22] D. Ehre, E. Lavert, M. Lahav, and I. Lubomirsky, Science 327, 672 (2010). 